\title{
Author Correction: TRPV1 SUMOylation regulates nociceptive signaling in models of inflammatory pain
}

Yan Wang1, Yingwei Gao', Quan Tian², Qi Deng¹, Yangbo Wang1, Tian Zhou¹, Qiang Liu², Kaidi Mei², Yingping Wang ${ }^{1}$, Huiqing Liu', Ruining Ma1, Yuqiang Ding³, Weifang Rong ${ }^{4}$, Jinke Cheng ${ }^{1}$, Jing $\mathrm{Yao}^{2}$, Tian-Le $\mathrm{Xu}{ }^{4}$, Michael $\mathrm{X} . \mathrm{Zhu}^{5} \&$ Yong $\mathrm{Li}^{1}$

Correction to: Nature Communications https://doi.org/10.1038/s41467-018-03974-7, published online 18 April 2018

In the originally published version of this Article, the affiliation details for Yan Wang, Yingwei Gao, Qi Deng, Yangbo Wang, Tian Zhou, Yingping Wang, Huiqing Liu, Ruining Ma, Jinke Cheng and Yong Li incorrectly omitted 'Shanghai Jiao Tong University'. This has now been corrected in both the PDF and HTML versions of the Article.'

Furthermore, the Supplementary Information file originally associated with this Article inadvertently omitted Supplementary Figure 9. The error has now been fixed and the corrected version Supplementary Information PDF is available to download from the HTML version of the Article.

Published online: 28 June 2018

Open Access This article is licensed under a Creative Commons Attribution 4.0 International License, which permits use, sharing, adaptation, distribution and reproduction in any medium or format, as long as you give appropriate credit to the original author(s) and the source, provide a link to the Creative Commons license, and indicate if changes were made. The images or other third party material in this article are included in the article's Creative Commons license, unless indicated otherwise in a credit line to the material. If material is not included in the article's Creative Commons license and your intended use is not permitted by statutory regulation or exceeds the permitted use, you will need to obtain permission directly from the copyright holder. To view a copy of this license, visit http://creativecommons.org/licenses/by/4.0/.
}

(C) The Author(s) 2018

\footnotetext{
${ }^{1}$ Department of Biochemistry and Molecular Cell Biology, Shanghai Key Laboratory for Tumor Microenvironment and Inflammation, Institute of Medical Sciences, Shanghai Jiao Tong University School of Medicine, Shanghai 200025, China. ${ }^{2}$ Hubei Key Laboratory of Cell Homeostasis, College of Life Sciences, Wuhan University, Wuhan, Hubei 430072, China. ${ }^{3}$ Department of Anatomy and Neurobiology, Collaborative Innovation Center for Brain Science, Tongji University School of Medicine, 200092 Shanghai, China. ${ }^{4}$ Department of Anatomy and Physiology, Shanghai Jiao Tong University School of Medicine, 200025 Shanghai, China. ${ }^{5}$ Department of Integrative Biology and Pharmacology, McGovern Medical School, The University of Texas Health Science Center at Houston, Houston, TX 77030, USA. Correspondence and requests for materials should be addressed to J.Y. (email: jyao@whu.edu.cn) or to Y.L. (email: liyong68@shsmu.edu.cn)
} 